\title{
Influenza A(H5N1) Virus Subunit Vaccine Administered with CaPNP Adjuvant Induce High Virus Neutralization Antibody Titers in Mice
}

\author{
Tulin Morcol, ${ }^{1,5}$ Peri Nagappan, ${ }^{2}$ Stephen J. D. Bell, ${ }^{3}$ and Andrew G. Cawthon ${ }^{4}$
}

Received 10 July 2019; accepted 9 September 2019; published online 7 October 2019

\begin{abstract}
The highly pathogenic avian influenza H5N1 virus continues to spread globally in domestic poultry with sporadic transmission to humans. The possibility for its rapid transmission to humans raised global fears for the virus to gain capacity for human-to-human transmission to start a future pandemic. Through direct contact with infected poultry, it caused the largest number of reported cases of severe disease and death in humans of any avian influenza strains. For pandemic preparedness, use of safe and effective vaccine adjuvants and delivery systems to improve vaccine efficacy are considered imperative. We previously demonstrated CaPtivate's proprietary $\mathrm{CaP}$ nanoparticles (CaPNP) as a potent vaccine adjuvant/delivery system with ability to induce both humoral and cell-mediated immune responses against many viral or bacterial infections. In this study, we investigated the delivery of insect cell culture-derived recombinant hemagglutinin protein (HA) of A/H5N1/ Vietnam/1203/2004 virus using CaPNP. We evaluated the vaccine immunogenicity in mice following two intramuscular doses of $3 \mu \mathrm{g}$ antigen combined with escalating doses of CaPNP. Our data showed CaPNP-adjuvanted HA(H5N1) vaccines eliciting significantly higher IgG, hemagglutination inhibition, and virus neutralization titers compared to non-adjuvanted vaccine. Among the four adjuvant doses that were tested, CaPNP at $0.24 \%$ final concentration elicited the highest IgG and neutralizing antibody titers. We also evaluated the inflammatory response to CaPNP following a single intramuscular injection in guinea pigs and showed that CaPNP does not induce any systemic reaction or adverse effects. Current data further support our earlier studies demonstrating CaPNP as a safe and an effective adjuvant for influenza vaccines.
\end{abstract}

KEY WORDS: CaP nanoparticles; adjuvant; A/H5N1; pandemic influenza.

\section{INTRODUCTION}

Influenza viruses are resilient in their ability to infect hundreds of millions of people annually and causing hundreds of thousands of hospitalizations and deaths among children and elderly. Vaccines are the most effective and economical countermeasure to prevent infectious diseases to save lives. The emergence of new variants of influenza viruses and occurrence of human infection outbreaks of avian influenza underscore the importance of accelerating vaccine development for pandemic preparedness. Both the US health agencies and $\mathrm{WHO}$ emphasize the need for new technologies for enhancing vaccine efficacy and to increase global

\footnotetext{
${ }^{1}$ CaPtivate Pharmaceuticals LLC, 3805 Old Easton Road, Doylestown, Pennsylvania 18902, USA.

${ }^{2}$ Clark Atlanta University, Atlanta, Georgia, USA.

${ }^{3}$ Proventus Bio Inc., Atlanta, Georgia, USA.

${ }^{4}$ Battelle, West Jefferson, Ohio, USA.

${ }^{5}$ To whom correspondence should be addressed. (e-mail: tmorcol@captivatepharma.com)
}

influenza vaccine capacity through the use of dose-sparing strategies $(1,2)$.

Avian influenza - the "bird flu"-is a virus that predominantly infects wild birds but can rapidly transmit to domestic poultry resulting in significant economic losses to the industry. Influenza $\mathrm{A} / \mathrm{H} 5 \mathrm{~N} 1$ is a highly pathogenic strain of avian influenza virus that has been shown to transmit to other animals and humans. H5N1 has caused the highest number of reported cases of severe disease and death in humans of any avian influenza viruses raising concerns of dissemination of a highly lethal influenza. According to the World Health Organization (WHO) reports, there have been 859 laboratory-confirmed cases and 453 deaths (53\% lethality) in humans in 2003-2017 (3). Although H5N1 has not been capable of sustaining human-to-human transmission, each additional case provides the virus opportunity to mutate and acquire the ability of viral adaptation $(4,5)$. The emergence of novel influenza virus strains and the threat of transmission to humans accelerated research towards developing universal influenza vaccines (UIV) that would ideally protect against all influenza infections or be broadly protective against multiple 
strains $(6,7)$. Yet, development of such a revolutionary strategy will unfortunately take many years and conflicts on approaches to develop a successful UIV exist $(8,9)$. Until a successful UIV becomes available, influenza vaccines eliciting broadly reactive immune responses with improved efficacy compared to existing vaccines remain essential. To this end, the WHO has recommended the development and stockpiling of monovalent influenza vaccines for all potential pandemic strains (10).

Currently available pandemic influenza vaccines result in poor immunogenicity in unprimed human populations $(11,12)$ requiring either large antigen doses or need administration with an appropriate adjuvant. There are two egg-grown, inactivated Influenza A/H5N1 virus vaccines that are approved by the Food and Drug Administration (FDA) for US stockpiling (13). Both vaccines are designed to contain squalene-based oil-in-water adjuvants, AS03 (by GSK) or MF59 (by Novartis). However, the potency of stockpiled vaccines may be severely limited given the anticipated antigenic drift/shift with the emergence of a novel strain of pandemic $\mathrm{H} 5 \mathrm{~N} 1$. In addition, inactivated vaccines containing approved adjuvants are still not as immunogenic as the live attenuated vaccines (14) (crossed referenced in 15).

During the H1N1(2009) pandemic, millions of doses of MF59- or AS03-adjuvanted vaccines have been used across all age groups, confirming superior immunogenicity and showed increased efficacy in young children against homologous or mismatched strains $(16,17)$. In preclinical studies, adjuvanted $\mathrm{H} 1 \mathrm{~N} 1$ pandemic vaccines showed cross protection against different strains of seasonal influenza viruses (18). However, the increased risks of narcolepsy reported among the children and adolescence vaccinated with A0S3adjuvanted H1N1 vaccine in 2009-2010 period raised serious concerns regarding the safety of squalene-based adjuvants (19-21). Although the underlying reasons to this increased risk remain unexplained and MF59 has not been associated to that risk, there are other factors that make oil-in-water emulsion adjuvants less than ideal. Adjuvants such as alum, MF59, or MPL predominantly indicate Th2-biased humoral responses but are limited in ability to induce $\mathrm{T}$ cell immunity (22,23). By enhancing adaptive immune responses, adjuvants may reduce the number of required immunizations and/or the amount of antigen needed to elicit a protective immune response. In addition, vaccine adjuvants may also provide longer-lasting protection and broader cross-protection against heterologous strains of pathogens. Federal agencies, including National Institute of Allergy and Infectious Diseases (NIAID), continue to urge discovery and development of new adjuvants that minimize the risk of systemic reactions while maximize vaccine efficacy and dose-sparing capacity.

Using particulate materials as vaccine adjuvants has gained transdisciplinary attraction for their ability to mimic pathogens that are commonly recognized, phagocytosed, and processed by APCs. An ideal nanocarrier that shows potential to improve vaccine efficacy should also provide protection to antigen against degradation, facilitate controlled release of the antigen, and, most importantly, must be safe for human use. Nanoparticle-based delivery systems can improve vaccine efficacy by protecting the vaccine antigen from degradation, increasing vaccine stability, eliciting delivery and sustain release of antigen to the specific sites, and facilitating antigen uptake and processing by antigen presenting cells (APCs) to achieve cellular immune responses (24).

In large number of preclinical studies, we and other investigators have shown that our calcium phosphate nanoparticles (CaPNPs) have the ability to enhance vaccine immunogenicity and efficacy and provide antigen dosesparing (25-32). In recent preclinical studies, we showed that an inactivated whole virus $\mathrm{H} 1 \mathrm{~N} 1$ pandemic influenza vaccine adjuvanted with CaPNP has capacity to significantly augment $\mathrm{VN}$ titers, increase protective efficacy, minimize inflammation and viral load in the lungs while allowing at least 10 -fold antigen dose-sparing (un-published data; reported to NIH and in a press release) (33). We have also demonstrated that protein/peptide molecules adsorbed to CaPNPs retain their structural and functional activity and are released in a sustained manner $(34,35)$.

We previously reported that an inactivated whole virus influenza A 2009 (H1N1pdm) (IIV) vaccine formulated with our calcium phosphate nanoparticles (CaPNPs) could significantly improve vaccine immunogenicity and efficacy and allow dose-sparing (22). In our most recent studies supported by the NIH/NIAID Animal Models of Infectious Diseases Preclinical Services Program, a single intramuscular (IM) administration of IIV-CaPNP vaccine at 1/10th of full dose induced significantly higher $\mathrm{IgG}$, hemagglutination inhibition (HAI), and virus neutralization (VN) titers than the full-dose non-adjuvanted vaccine and provided nearly full protection against a lethal dose of homologous virus challenge [unpublished data]. Our findings suggested that CaPNP-adjuvanted vaccine neutralized the virus quickly and efficiently before a strong (and potentially pathogenic) immune response was triggered. These findings supported our previous suggestions that CaPNP adjuvant action must elicit both humoral and cellular immune responses to induce protection against influenza virus infections (25).

Antigen presenting cells (APCs), particularly the dendritic cells (DCs), are the focus of most vaccine development strategies because of their unique antigen-presenting potential and the ability to induce cellular immune responses (36). We have shown that CaPNP indicates distinctive properties as delivery system to efficiently deliver antigens to APCs and directly activate the maturation of DCs to generate a robust cytotoxic T cell (CTL) response (37). That was indicated by upregulation of co-stimulatory molecules and MHC class II molecules to generate a robust CD8+ CTL response. The increased innate cell activity and the magnitude of CD8+ CTL response induced by CaPNP were comparable to the response induced by Montanide, a water-in-oil emulsion known to be the most potent inducer of CTL responses (37). The ability of our CaPNP to induce strong $\mathrm{T}$ cell responses has also been demonstrated independently by others $(26,30,31)$.

Majority of approaches to develop protective immunity against influenza infection aim to induce high levels of antibodies against HA protein. As such, the HA protein has been the antigen of choice for development of vaccines against $\mathrm{H} 5 \mathrm{~N} 1$ viruses. However, subunit vaccines often indicate weak immunogenicity than the whole organism vaccines thus require the use of an adjuvant to augment vaccine effectiveness. In previous clinical studies, recombinant HA(H5N1) subunit vaccines at $90 \mu \mathrm{g}$ HA dose administered with prime-boost schedule induced only modest 
responses $(11,12)$. These findings prompted the use of adjuvants in the vaccine. Currently, the leading $\mathrm{H} 5 \mathrm{~N} 1$ vaccine adjuvants are oil-in-water $(\mathrm{o} / \mathrm{w})$ emulsions that augment neutralizing antibody titers, increase the breadth of crossreactive antibodies, and also possess dose-sparing activity (38-40). MF59 is a squalene-based oil-in-water emulsion which became the first novel adjuvant approved in 2015 for use in seasonal influenza vaccines (e.g., FLUAD), which was after 80 years of approval of aluminum-based adjuvants $(41,42)$.

In this report, we describe formulation of a subunit A/ H5N1 vaccine composed of recombinant HA protein from a wild-type influenza A/H5N1/Vietnam/1203/2004 strain, grown in insect cells, adsorbed to CaPNPs as the adjuvant. We evaluated the vaccine immunogenicity in mice with respect to induction of antigen-specific IgG titers and virus neutralization antibody titers after two intramuscular (IM) doses of $3 \mu \mathrm{g}$ HA containing varying adjuvant doses administered at a prime-boost schedule. In "Discussion" section, we also discuss some advantages of CaPNP over oil-in-water emulsion adjuvants, such as MF59.

In previous preclinical safety/toxicity studies, CaPNPs administered by intradermal (ID) or subcutaneous (SC) routes in mice (37), intravaginally (IVag) in mice (28), or by inhalation routes in guinea pigs (34) has induced no systemic or local adverse effects, toxicity, or inflammatory responses. Also, in a phase 1 double-blind placebo-controlled human clinical trial conducted in human subjects in the US, CaPNP administered SC indicated no adverse effects, toxicity, or inflammatory responses, or no skin irritation at the injection side (un-published data reported to U.S. Food and Drug Administration (FDA); disclosed in press releases) (43). In this article, we describe acute toxicity studies of CaPNP in guinea pigs following IM injection as the conventional route of influenza vaccine administration.

\section{MATERIALS AND METHODS}

\section{Ethics Statement}

All immunization studies were performed at BioCon Inc. (Rockville, MD) in the company's Association for Assessment and Accreditation of Laboratory Animal Care International (AAALAC)-accredited Laboratory Animal Research Facility. Animals were housed according to Office of Laboratory Animal Welfare (OLAW) and AAALAC Guidelines. All animal experiments and related protocols were reviewed and approved by the Institutional Animal Care and Use Committee (IACUC) of BioCon. Experiments were conducted in accordance with the guidelines set out by the AAALAC. Acute toxicity studies were performed separately with contract to IIT Research Institute (IITRI-Chicago, IL.) in accordance with U.S. Food and Drug Administration (FDA) Good Laboratory Practice (GLP) Regulations as set forth in the Code of Federal Regulations.

\section{Virus Products and Reagents}

Full length glycosylated recombinant HA protein of A/H5N1/Vietnam/1203/2004 strain (referred as "HA" or HA(H5N1) throughout the text) was purchased from Protein
Sciences (Meriden, CT). The protein was produced in insect cells using the baculovirus expression vector system and characterized by the vendor as $83 \mu \mathrm{g} \mathrm{HA} / \mathrm{ml}$ with $>90 \%$ purity. Protein Assay kit was purchased from BioRad (Hercules, CA). All antibodies and ABTS peroxidase substrate used in ELISA were purchased from Kirkegaard and Perry Laboratories (Gaithersburg, MD). Influenza A/H5N1 positive antibody reference serum for ELISA was obtained from the Centers for Disease Control and Prevention (CDC) (Atlanta, GA). HAI and VN assays were performed by Battelle Biomedical Research Center (West Jefferson, OH) according to institutional Standard Operating Procedures (SOPs). Receptor destroying enzyme (RDE) was obtained from Accurate Chemical and Scientific (Westbury NY). Horse erythrocytes and bovine serum albumin (BSA) were from Lampire Biological Laboratories (Pipersville, PA). The challenge virus solution composed of highly pathogenic avian influenza A/H5N1/VN1203 (VN1203) propagated in MadinDarby Canine Kidney (MDCK) tissue culture used in the microneutralization assay was product of Battelle Biomedical Research Center (West Jefferson, $\mathrm{OH}$ ).

\section{Vaccine Formulation}

CaPNPs were manufactured using our proprietary process. Briefly, inorganic salt solutions of calcium chloride, sodium citrate, and dibasic sodium phosphate were mixed at predetermined ratios to precipitate calcium phosphate. The ratios of inorganic salt solutions were adjusted to obtain particles with mean particle sizes of $200 \mathrm{~nm}$. The particle size was determined by photon correlation spectroscopy using Coulter N4Plus submicron particle sizer. Vaccines containing $3 \mu \mathrm{g}$ HA per $100 \mu \mathrm{l}$ dose with or without CaPNP adjuvant were formulated as described elsewhere $(25,27)$. Four different concentrations of CaPNP in $0.03-0.24 \%$ (weight/volume) range were evaluated.

\section{HA Adsorption Efficiency to CaP Nanoparticles}

The amount of HA protein adsorbed to CaP nanoparticles was determined similar to described elsewhere $(34,35)$. Briefly, triplicate samples from $\mathrm{HA}+\mathrm{CaPNP}$ formulations containing $30 \mu \mathrm{g} / \mathrm{ml} \mathrm{HA}(\mathrm{H} 5 \mathrm{~N} 1)$ antigen either in $0.03 \%$, $0.06 \%, 0.12 \%$, or $0.24 \% \mathrm{CaPNP}$ were centrifuged at $4000 \times g$ for $15 \mathrm{~min}$ at $4^{\circ} \mathrm{C}$ to separate bound protein in the particle pellet from the free protein in supernatant. The pellets were washed twice in sterile water and then dissolved in $0.01 \mathrm{~N}$ $\mathrm{HCl}$ to elute the protein that was initially adsorbed to CaPNPs. The amount of total protein in solubilized pellets and wash fractions were determined by Bradford's method using BioRad Protein Assay. The standard curve for the assay was prepared by serial 1:2 dilutions of purified HA(H5N1) protein in the $5-40 \mu \mathrm{g} / \mathrm{ml}$ concentration range. Samples taken directly from the original HA + CaPNP particulate suspension were also included in the assay as total protein control for material balance. All test samples, controls, and standard dilutions used in BioRad assay were applied in 96-well plates in triplicates. We should note that particulate suspensions of HA + CaPNPs at concentrations used here are $100 \%$ soluble in the acidic dye reagent of the assay thus can directly be added to the wells to quantify and confirm the total protein in the formulation (34). The percentage of HA adsorption 
efficiency ( $\%$ AE) of CaPNP was calculated using the following equation:

$\% \mathrm{AE}=[\mathrm{HA}$ in solubilized pellet $] /[$ total $\mathrm{HA}$ originally added in vaccine $] \times 100$

\section{Immunization and Sample Collection}

In vivo studies were performed at BioCon Laboratory Animal Research Facility. Immunizations and sample collections were performed in accordance with the approval of the IACUC. Female BALB/c mice at the age of 6-8 weeks were purchased from Harlan Sprague Dawley Inc. (currently Envigo, Madison, WI). CaPNP-adjuvanted vaccines containing a fixed dose of HA in varying doses of adjuvant were administered without separating bound HA on particles from free HA in solution. Given that the currently approved human influenza vaccines are administered IM, we used the same route in this study for its relevancy to product development. As shown in Table I, five groups of 10 mice were immunized with $3 \mu \mathrm{g}$ non-adjuvanted or CaPNPadjuvanted HA vaccine as two $50-\mu 1$ injections into each hind limbs. Each mouse received a primary inoculation and a booster dose at 2-week intervals. Additional two groups of 10 mice were administered CaP only (at highest concentration) as the placebo control or kept un-immunized as negative control. At 2-week post primary immunization and prior to the booster dose, blood samples from all mice in each group were collected via the orbital sinus. Equal volumes of serum from individual mouse per each group were pooled and stored at $-80^{\circ} \mathrm{C}$. Blood collection continued at 2-week intervals for 10 weeks for monitoring of HA(H5N1)-specific antibody responses by ELISA. Serum samples from group 2 (0.06\% CaPNP) and group $4(0.24 \%$ CaPNP) at 10 -week post primary immunization were used for determination of HAI and VN antibody titers as described below.

\section{Enzyme-Linked Immunosorbent Assay (ELISA): Adjuvant Dose-Response}

The optimal adjuvant concentration in the vaccine was assessed with respect to induction of $\mathrm{HA}(\mathrm{H} 5 \mathrm{~N} 1)$-specific $\mathrm{IgG}$ responses by indirect ELISA. Briefly, multiples of microtiter plates were coated with $100 \mathrm{ng} /$ well recombinant baculovirusderived HA(H5N1) protein diluted in phosphate-buffered saline (PBS) and incubated overnight at $4{ }^{\circ} \mathrm{C}$. Subsequent to washing and blocking with PBS plus $0.05 \%$ tween-20 (PBST), serial 1:2 dilutions of immune sera and negative controls in triplicates were applied to designated wells in 96-well plates in duplicate. Following incubation with HRP-conjugated secondary anti-mouse IgG antibody and subsequent washing, ELISA was developed using ABTS peroxidase substrate and the absorbance was read at $405 \mathrm{~nm}\left(\mathrm{~A}_{405}\right)$. The cut-off value for the assay was defined as 4 times the mean absorbance value of a negative control serum. The absorbance readings equal or greater than the cut-off value were considered positive in determining the endpoint titers.

\section{Hemagglutination Inhibition Assay (HAI)}

Pooled serum samples from the vaccine group inducing the highest IgG titers at all time points (i.e., group 4: HA + $0.24 \% \mathrm{CaP}$ ) and an additional group inducing lower $\mathrm{IgG}$ titers (i.e., group 2: $\mathrm{HA}+0.06 \% \mathrm{CaP}$ ) were assayed for virusneutralizing antibody tiers. Briefly, serum samples were collected at 10 -week post first immunization from the groups immunized twice with $3 \mu \mathrm{g}$ HA, either in $0.06 \%$ or $0.24 \%$ CaPNP. Pooled sera per group were pre-treated with receptor-destroying enzyme (RDE) from vibrio cholerae (1 part sera +3 parts $\mathrm{RDE}$ ) for $18 \mathrm{~h}$ at $37^{\circ} \mathrm{C}$ prior to heat inactivation at $56^{\circ} \mathrm{C}$ for $30 \mathrm{~min}$. Samples were allowed to cool to room temperature and then serially diluted in PBS in Vbottom microtiter plates in quintuplicate (i.e., 5 replicates per/ sample/dilution). An equal volume of 8 hemagglutination units (HAU) of VN1203 virus was added and incubated at room temperature for $1 \mathrm{~h}$. Finally, 1\% horse erythrocytes in PBS plus $0.5 \%$ BSA was added. The HAI titers were determined after $30 \mathrm{~min}$ by tilting the plates and assessing the endpoint titer for each sample. The HAI titers are reported as the reciprocal of the highest serum dilution at which hemagglutination was completely inhibited.

\section{Microneutralization Assay (MN)}

The microneutralization assay is a cell-based assay that detects the presence of virus-neutralizing antibodies (VN) in a sample by utilizing the antibodies' ability of inhibiting viral particles to infect healthy cells thus preventing cytopathic effect (CPE). On the day prior to $\mathrm{MN}$ assay, 96-well microtiter plates were seeded with MDCK cells at $3.0 \times 10^{5}$ cells/well in maintenance media (Eagle's Minimum Essential Medium (EMEM), sodium pyruvate, L-glutamine, and Penicillin-Streptomicin) containing $10 \%$ fetal bovine serum (FBS). The plates were incubated at $37^{\circ} \mathrm{C}, 5 \% \mathrm{CO}_{2}$ for $16-$ $24 \mathrm{~h}$ to obtain $\geq 90 \%$ confluent cell monolayer at the time of the assay. On the day of the experiment, pooled sera from groups 2 and 4 (Table I) were heat inactivated at $56^{\circ} \mathrm{C}$ for $30 \mathrm{~min}$ and 10-point dilution series were prepared in serumfree media. Approximately $600 \mathrm{TCID}_{50} /$ sample of challenge virus was added in all sample dilutions, positive control (PC; $\left.2.47 \times 10^{4} \mathrm{ND}_{50} / \mathrm{ml}\right)$, and the negative control (NC), except the virus control (VC). After incubation at $37^{\circ} \mathrm{C}, 5 \% \mathrm{CO}_{2}$ for $1 \mathrm{~h}$, each sample dilution plus virus was transferred to the washed cell-seeded 96-well plates in quintuplicate and incubated at $37^{\circ} \mathrm{C}, 5 \% \mathrm{CO}_{2}$ for 4 days. The plates were inspected for the presence or absence of CPE. The neutralizing titer $\mathrm{ND}_{50}$ was defined as the reciprocal of antiserum dilution that results in the absence of CPE in $50 \%$ of the wells inoculated (i.e., virus growth $50 \%$ inhibited). The $\mathrm{ND}_{50}$ values were calculated by the number of the virus negative 
Table I. Vaccine Composition and Groups

\begin{tabular}{|c|c|c|c|c|c|}
\hline \multirow[t]{2}{*}{ Group No. } & \multirow[t]{2}{*}{ Number of mice } & \multirow[t]{2}{*}{ Vaccine } & \multicolumn{2}{|c|}{ Adjuvant/dose (CaPNP) } & \multirow[t]{2}{*}{ Antigen/dose ( $\mu \mathrm{g} \mathrm{HA})$} \\
\hline & & & $\mu \mathrm{g}$ & $\%$ & \\
\hline 1 & 10 & $\mathrm{HA}+0.03 \% \mathrm{CaP}$ & 30 & 0.03 & 3 \\
\hline 2 & 10 & $\mathrm{HA}+0.06 \% \mathrm{CaP}$ & 60 & 0.06 & 3 \\
\hline 3 & 10 & $\mathrm{HA}+0.12 \% \mathrm{CaP}$ & 120 & 0.12 & 3 \\
\hline 4 & 10 & $\mathrm{HA}+0.24 \% \mathrm{CaP}$ & 240 & 0.24 & 3 \\
\hline 5 & 10 & HA alone & - & - & 3 \\
\hline 6 & 10 & $\mathrm{CaP}$ alone & 240 & 0.24 & 0 \\
\hline 7 & 10 & Un-immunized & - & - & - \\
\hline
\end{tabular}

wells and serum dilution using the Spearman Kärber statistical method (44). The acceptance criteria for the MN assay was at least 5 of the cell culture control wells must be a healthy and $\geq 90 \%$ confluent monolayer at the end of the experiment, the $\mathrm{ND}_{50}$ titer of the PC must be $\pm 0.5 \log _{10}$ of its certified titer, NC should not display signs of neutralization, and virus back titration should result in approximately $2 \times 10^{3}$ $\mathrm{TCID}_{50} / \mathrm{ml}$.

\section{Acute Toxicity of Inflammatory Response to Intramuscularly Administered CaPNP}

This study was conducted separately and in accordance with FDA Good Laboratory Practice (GLP) regulations to evaluate any acute toxicity and site-specific inflammatory response risk of IM-administered CaPNPs in a guinea pig model. Three groups of 5 male and 5 female CRL:(HA)BR Hartley albino guinea pigs, 3-4 weeks of age, were administered a single dose of approximately $0.5 \mathrm{mg}$ CaPNP $(\sim 1.2 \mathrm{mg}$ $\mathrm{CaPNP} / \mathrm{kg}$ body weight) in two IM injections to the right femoris muscle of the hind limb. A fourth group served as the untreated control. Animals were observed daily for 14 days for any adverse clinical signs, injection site inflammation, and change in body weight. Hematology parameters and histopathology of designated tissues were evaluated in all pathology animals designated for terminal sacrifice. Hematology parameters included white blood cell count, platelet count, erythrocyte count, erythrocyte morphology, and others. Limited diagnostic pathology consisted of collection and evaluation of the injection site tissues and site specific draining lymph nodes. Bone marrow, spleen, and thymus were also collected in all groups for examination of the inflammatory response. The tissues for histopathology and all gross lesions were examined microscopically. Any lesions or abnormal appearance were documented. Any changes received a severity score based on the following scale: $0=$ no change, $1=$ minimal, $2=$ mild, $3=$ moderate, and $4=$ marked. Final average severity scores for each change in each tissue sample were slightly modified by weighting factors automatically assigned by the LABCAT $®$ histopathology data management program (IPA, Princeton, NJ) built-in system. Thus, the weighing averages are assigned based upon the "severity" and "distribution" as outlined in the following table.

\begin{tabular}{lllll}
\hline Severity Grading & No Modifier & Focal & Multifocal & Diffuse \\
\hline 0 & 0 & - & - & - \\
1 & 1.0 & 0.25 & 0.5 & 0.75 \\
2 & 2.0 & 1.25 & 1.5 & 1.75 \\
3 & 3.0 & 2.25 & 2.5 & 2.75 \\
4 & 4.0 & 3.25 & 3.5 & 3.75 \\
\hline
\end{tabular}

\section{Statistical Analysis}

Hematology data were statistically analyzed by ANOVA followed by Dunnett's test, using LABCAT®. The enzymatic and cellular data were statistically analyzed by $t$ test using Systat. ELISA data were analyzed using ANOVA followed by Tukey's post hoc test for multiple comparisons. A " $p$ " value of $<0.05$ was considered statistically significant in all comparisons. HAI and VN data could not be analyzed by ANOVA due to absence of standard deviation between the quantiplicates of pooled sera used in the assays.

\section{RESULTS}

\section{Adsorption Efficiency of HA to CaPNP}

The amount of HA adsorbed to CaPNP was quantified using the BioRad protein assay. HA + CaPNPs particles were collected by centrifugation and the bound HA protein was eluted by solubilizing the particle pellets in $0.01 \mathrm{~N} \mathrm{HCl}$. We previously established that neither $\mathrm{HCl}$ nor solubilized components of calcium phosphate interferes with the dye reaction in the assay $(34,35)$. The total protein in pellet fractions was calculated from the standard curve prepared by serial dilutions of purified $\mathrm{HA}(\mathrm{H} 5 \mathrm{~N} 1)$ as the protein reference, rather than bovine serum albumin used in conventional methods. Since HA is the only protein component in the formulation, this allows us quantification of the protein adsorbed to CaPNP with accuracy comparable to ELISA methods $(34,35)$.

As shown in Table II, there was no significant difference between the total amount of HA protein initially added based 
Table II. HA(H5N1) Adsorption Efficiency of CaPNP

\begin{tabular}{|c|c|c|c|c|}
\hline \multirow[t]{2}{*}{ Vaccine formulation } & \multicolumn{2}{|c|}{ Total protein $(\mathrm{HA})(\mu \mathrm{g} / \mathrm{ml})$} & \multirow[t]{2}{*}{ Total protein $(\mu \mathrm{g} / \mathrm{ml})$ adsorbed to CaPNP by BioRad Assay (c) } & \multirow[t]{2}{*}{$\% \operatorname{AE}(d)$} \\
\hline & Theoretical $^{\mathrm{a}}$ & By BioRad Assay (b) & & \\
\hline $\mathrm{HA}+0.03 \% \mathrm{CaP}$ & 30 & $29.2 \pm 0.2$ & $24.1 \pm 0.4$ & 83 \\
\hline $\mathrm{HA}+0.06 \% \mathrm{CaP}$ & 30 & $30.5 \pm 0.4$ & $28.4 \pm 0.6$ & 93 \\
\hline $\mathrm{HA}+0.12 \% \mathrm{CaP}$ & 30 & $29.5 \pm 0.4$ & $29.2 \pm 0.7$ & 100 \\
\hline $\mathrm{HA}+0.24 \% \mathrm{CaP}$ & 30 & $30.2 \pm 0.6$ & $29.8 \pm 0.4$ & 100 \\
\hline
\end{tabular}

${ }^{a}$ Amount of HA originally added (based on vendor product info)

${ }^{b}$ As determined by BioRad assay in aliquots taken directly from CaPNP+HA(H5N1) particulate suspensions. Values as mean of triplicates $( \pm \mathrm{SD})$

(c)Amount of HA in solubilized pellets measured by BioRad assay. Values as mean of triplicates $( \pm \mathrm{SD})$

(d)Percent adsorption efficiency calculated from the ratio of HA in pellet to total HA originally added; both as measured by the BioRad assay (also see Eq. 1). Values rounded to the nearest whole number

on the vendor's specifications ("theoretical" amount) vs the amount of protein "calculated" in the formulation after dissolving the HA + CaPNP in BioRad assay's dye reagent. In most $\mathrm{HA}+\mathrm{CaPNP}$ formulations, $>90 \%$ of $\mathrm{HA}$ was adsorbed to particles. In the formulations containing $0.12 \%$ or $0.24 \%$ CaPNP, essentially all HA initially added in the formulation was adsorbed to CaPNP.

\section{HA(H5N1)-Specific ELISA Titers: Adjuvant Dose-Response}

We evaluated the magnitude of $\mathrm{HA}(\mathrm{H} 5 \mathrm{~N} 1)$-specific antibody response in mice following two IM administration with $\mathrm{HA}(\mathrm{H} 5 \mathrm{~N})+\mathrm{CaPNP}$ vaccines containing $3 \mu \mathrm{g}$ per dose $\mathrm{HA}$ alone or with escalating doses CaPNP in the 0.03 to $0.24 \%$ range. Serum was drawn at 2 -week intervals for 10 weeks. Pooled sera from each group were assayed by HA(H5N1)-specific ELISA. After the antigen-antibody reaction in ELISA plates were developed and read at $\mathrm{A}_{405} \mathrm{~nm}$, the $\log _{2}$ geometric means of the reciprocal of sera dilutions vs $\mathrm{A}_{405}$ values for each group were plotted. The positive antigen-specific IgG titer was defined as the highest serum dilution that indicates absorbance value 4-fold greater than that of non-immune sera. The end point titers were calculated from the $\mathrm{A}_{405}$ vs $\log _{2}$ graphs.

Figure 1 shows the $\log _{10}$-transformed adjuvant doseresponse ELISA titers. The antibody response in all groups increased significantly after the booster immunization on day 14. With the exception of $\mathrm{HA}+0.03 \% \mathrm{CaPNP}$, all other CaPNP-adjuvanted vaccines at all time points induced significantly higher HA(H5N1)-specific ELISA titers than the non-adjuvanted vaccine which were statistically significant $(p<0.0001)$. The vaccine containing $0.24 \%$ CaPNP induced the highest IgG titers at all sampling points during the 10 -week evaluation. ELISA data suggested a positive correlation between the amount of CaPNP adjuvant in the vaccine and the serum antibody response.

\section{Hemagglutination Inhibition (HAI) Antibody Titers}

Figure 2 shows $\log _{10}$-transformed virus-specific HAI titers in pooled sera from 10 mice/group at week 10 post immunization with two IM dose of $3 \mu \mathrm{g} H A(H 5 N 1)$, alone or either with $0.06 \%$ or $0.24 \%$ CaPNP. Both the non-adjuvanted and CaPNP-adjuvanted vaccines indicated HAI titers of $\geq 160$ (Table IV). The vaccines formulated with $0.06 \%$ or $0.24 \%$ CaPNP showed significantly higher HAI titers (2-fold and 4-fold higher, respectively) compared to the nonadjuvanted vaccine. The HAI titers induced by the CaPNPadjuvanted vaccines appeared to correlate with the amount of CaPNP adjuvant present in the vaccine. The highest adjuvant content (i.e $0.24 \%$ ) induced the highest HAI titer. The vaccine inducing the highest $\mathrm{IgG}$ titers (i.e., $\mathrm{HA}+0.24 \%$ CaPNP) (Fig. 1) also induced the highest HAI titer (Fig. 2).

\section{Virus Neutralization Antibody Titers}

Virus neutralization antibody titers, shown in Fig. 3, followed a similar pattern with that of HAI titers (see Fig. 2) but with no direct correlation in magnitudes (see Table IV). The vaccine containing $0.24 \% \mathrm{CaPNP}$ induced the highest $\mathrm{VN}$ titers expressed in terms $\log _{10} \mathrm{ND}_{50} . \mathrm{ND}_{50}$ was defined as the concentration of anti-virus antibody that reduced the number of infected cells by $50 \%$. VN titers in the $\mathrm{HA}+0.24 \% \mathrm{CaPNP}$ vaccine group were significantly higher (nearly 5-fold) than the non-adjuvanted vaccine. VN titer of $\mathrm{HA}+0.24 \%$ CaPNP group was also 2.6-fold higher than the vaccine adjuvanted with $0.06 \%$ CaPNP.

\section{Acute Toxicity of Inflammatory Response to Intramuscularly Administered CaPNP}

A summary of most important observations from acute toxicity study is shown in Table III. In the study, a single IM dose of $1.2 \mathrm{mg} \mathrm{CaPNP} / \mathrm{kg}$ was administered in male and female Guinea pigs. Multiple clinical, pathological, and hematology parameters were evaluated at 14 days post-treatment. During the 14-day observation, all animals that received CaPNP appeared normal and no biologically significant clinical observations different than the untreated controls were made. All animals in the CaPNP-treated or untreated control groups gained weight at similar rates. There was no notable change in hematology values or morphology of red blood cells (RBCs) or platelets. No noteworthy gross lesions were observed at necropsy. The only lesion recorded was a slight pigmentation in the Salivary Gland of one Guinea pig which was considered incidental and not related to CaPNP. In the CaPNP treatment groups, a total of 6 out of 10 animals 


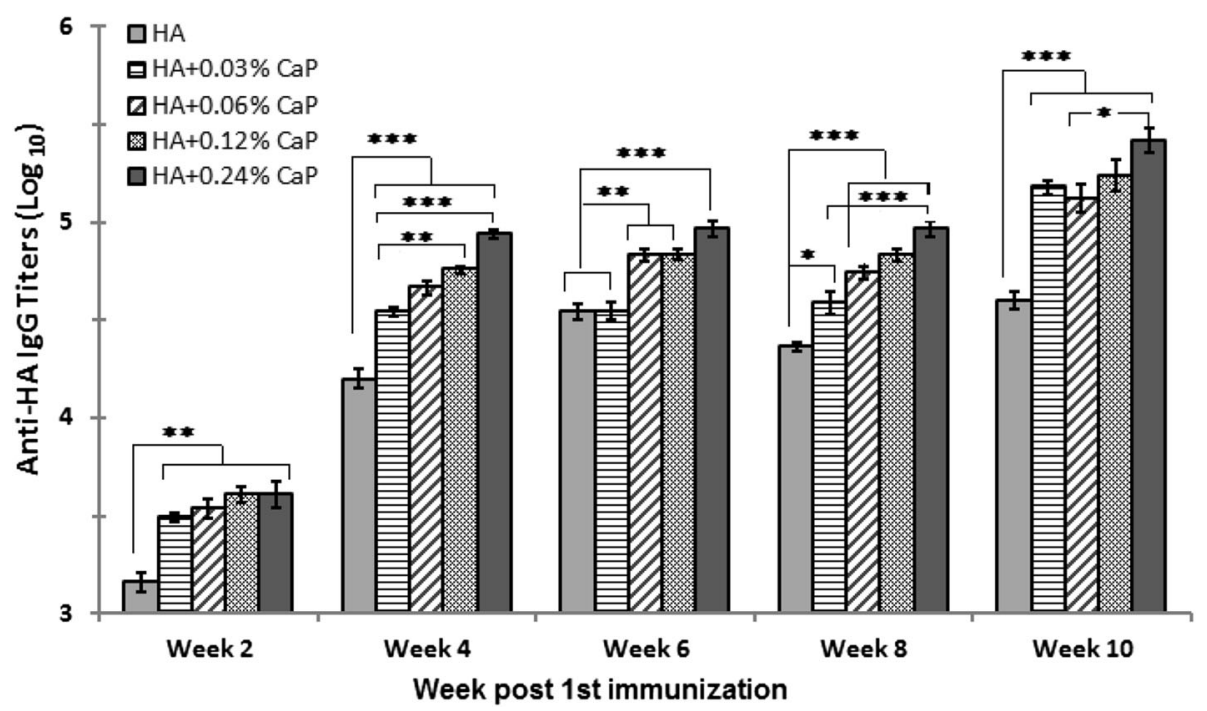

Fig. 1. HA-specific serum antibody response by ELISA following immunization with two i.m. doses of $3 \mu \mathrm{g} \mathrm{HA}(\mathrm{H} 5 \mathrm{~N} 1), 2$ weeks apart, alone or with escalating doses of CaPNP adjuvant. All values are expressed as log-transformed geometric mean with respected standard error of the mean (calculated $\log _{10}$ base) of triplicates of pooled sera per group from two experiments. Cut-off value for the assay was defined as 4 times the mean absorbance value of a negative control serum. CaP placebo readings at all time points were below the cut-off value and comparable to negative control thus not shown in the figure. Statistically significant differences were tested by ANOVA of logtransformed antibody titers for all vaccine groups at each time point ( $p \leq 0.0003$ for all). Multiple comparisons were tested by ANOVA/Tukey's post hoc. Significant differences between groups are linked by brackets $(* p<0.05, * * p<0.01, * * * p<0.001)$. Multiple groups indicating same degree of significant difference are collectively linked under single bracket for practical/visual purposes

showed minimal Granulomatous inflammation, with severity score 1 or lower on $0-4$ scale, in the injection site skeletal muscle. The lesions were focal and consisted of macrophages and small number of other inflammatory cells. No other CaPNP-related lesions were observed (see Method section "Acute Toxicity of Inflammatory Response to Intramuscularly Administered CaPNP" for description of scoring).

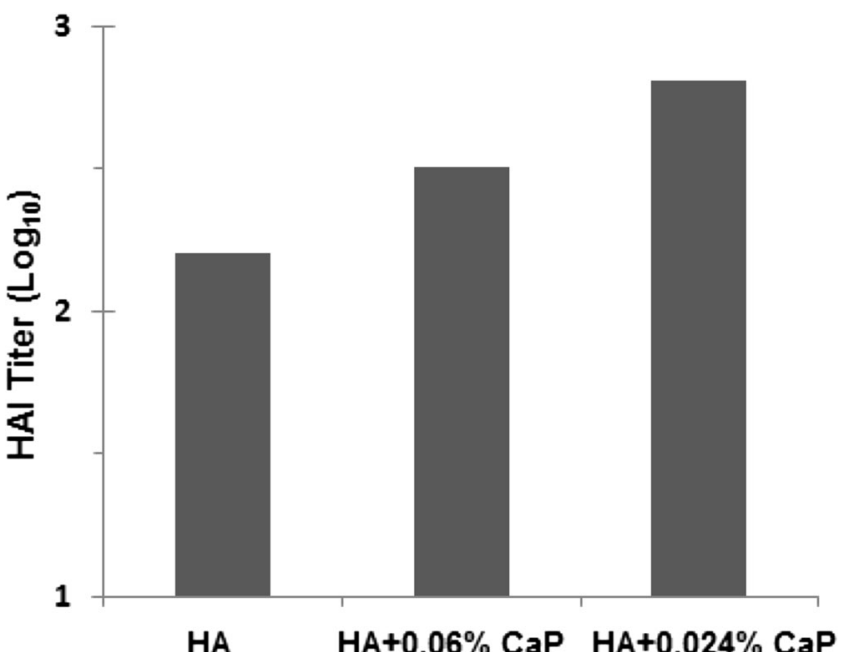

Fig. 2. Hemagglutination inhibition titers following vaccination with two i.m. doses of $3 \mu \mathrm{g} \mathrm{HA}(\mathrm{H} 5 \mathrm{~N} 1)$ vaccine either with $0.06 \%$ or $0.24 \%$ CaPNP or without the adjuvant. Bars represent the log-transformed geometric means of pooled sera (in quintuplicates) at week 10 post 1 st immunization

\section{DISCUSSION}

In this 10 -week study, we evaluated adjuvant effect of CaPNPs in a subunit H5N1 pandemic influenza vaccine HA protein antigen produced in insect cells. We determined vaccine immunogenicity with respect to antigen-specific IgG titers and virus-specific HAI and VN titers after prime-boost immunization with $3 \mu \mathrm{g} \mathrm{HA}(\mathrm{H} 5 \mathrm{~N} 1)$, alone or containing varying doses of CaPNP. The data we presented here suggest that $\mathrm{H} 5 \mathrm{~N} 1$ subunit vaccines adjuvanted with CaPNPs possess significantly higher immunogenicity than the vaccine without the adjuvant. Among the CaPNP doses tested, 0.24\% appeared to enhance vaccine immunogenicity substantially higher than the adjuvant doses lower than $0.24 \%$. This is mostly consistent with our previous findings (published and un-published) indicating that CaPNP at about $0.3 \%(w / v)$ was optimal for most vaccine antigens (25,37). Adjuvanting HA(H5N1) with CaPNP significantly augmented the antigen-specific $\mathrm{IgG}$ responses and boosted the HAI and VN titers, nearly 5-fold at $0.24 \%$ CaPNP dose compared to the non-adjuvanted vaccine control (see tabulated summary in Table IV). Our findings here and in our previous studies are also supported by large number of vaccine studies conducted, independently, by other research teams by reproducing and citing our nanoparticles and proprietary protocols. In one of these studies highly relevant to avian infections with potential to infect humans, a commercially available live Newcastle Disease Virus (NDV) vaccine was co-formulated with CaPNP and administered, at doses based on the live virus HA activity, in chickens by intranasal or intraocular routes (30). Newcastle disease is considered as 


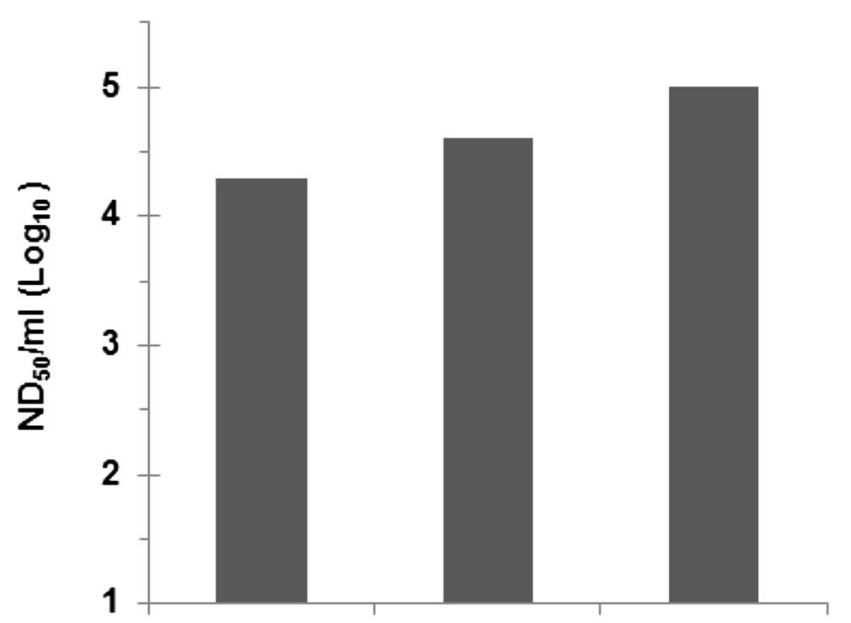

HA $\quad \mathrm{HA}+0.06 \% \mathrm{CaP} \quad \mathrm{HA}+0.024 \% \mathrm{CaP}$

Fig. 3. Virus neutralizing antibody titers per unit volume following two i.m. doses of $3 \mu \mathrm{g} \mathrm{HA}(\mathrm{H} 5 \mathrm{~N} 1)$ either with $0.06 \%$ or $0.24 \%$ CaPNP or without the adjuvant. The $50 \%$ neutralization dose $\left(\mathrm{ND}_{50}\right)$ is defined as the concentration of anti-virus antibody that reduced the number of infected cells by $50 \%$. Bars represent the log of geometric means of neutralization titers of pooled sera at week 10 post 1 st immunization

one of the two most serious diseases affecting poultry and other birds, the other being the highly pathogenic avian influenza H5N1. Authors reported that CaPNP-adjuvanted NDV vaccine elicited prolonged serum HAI and ELISA titers even at 4 and 5 weeks post-vaccination, while the titers in commercial vaccine group declined to insignificant levels at those time points. CaPNP-NDV vaccine also elicited strong cell-mediated immunity and mucosal $\operatorname{IgA}$ responses. In a report published by $\mathrm{CDC}$ evaluating the "validity of mouse as a mammalian model to study the H5N1 influenza virus pathogenesis and immunity", mice were immunized primeboost with various $\mathrm{H} 5 \mathrm{~N} 1$ vaccine formulations at $3 \mu \mathrm{g}$ HA per dose (45). It was shown that serum HAI antibody titers of 40 were sufficient to protect the mice from lethal challenge with $\mathrm{H} 5 \mathrm{~N} 1$ virus. All mice $(100 \%)$ with serum HAI titers of $\geq 80$ and $93 \%$ of mice with HAI titers of 40 were protected from death following challenge infection with a homologous virus. On the other hand, $100 \%$ of mice with HAI titers of $\leq 20$ died within 7 days of infection. Although our current study did not involve virus challenge, the HAI titers we report in Table IV and Fig. 2 compared with the CDC study strongly suggest that potentially $100 \%$ of mice in all CaPNP vaccine groups might have also been protected from a challenge infection. The HAI titers induced by the CaPNPadjuvanted vaccines were 8-16-fold higher than the generally accepted HAI threshold of 40. As we have also shown in previous studies (25), this suggests that CaPNP-adjuvanted $\mathrm{H} 5 \mathrm{~N} 1$ vaccine could potentially provide full protection even at lower than $3-\mu \mathrm{g}$ vaccine doses by allowing dose sparing. Dose sparing property of an adjuvant is particularly important for the expansion of national and global vaccine production capacities in the event of an influenza emergency.

In another $\mathrm{H} 5 \mathrm{~N} 1$ vaccine study, authored by Wong et al. (46), ferrets were immunized prime-boost with an inactivated A/H5N1/Vietnam/1203/2004 virus vaccine (i.e., the same strain used in our study) containing $7.5 \mu \mathrm{g}$ HA with MF59 or A0S3 as adjuvant and vaccine immunogenicity was evaluated. While the serum HAI in non-adjuvanted vaccine groups was undetectable, MF59-adjuvanted vaccine increased HAI titers in the $\log _{10} 2.2-2.7$ ranges. However, neither MF59- nor A0S3-adjuvant elicited any detectable VN titers. It was concluded that inactivated $\mathrm{H} 5 \mathrm{~N} 1$ vaccine had poor capacity to induce virus neutralizing antibodies. While we acknowledge the differences between our study and Wong et al. (e.g., different animal model) to make any direct comparison to our current results, it is still important to note that the HAI titers we achieved with only $3 \mu \mathrm{g}$ HA dose of CaPNP-adjuvanted H5N1 vaccine are higher (i.e., 320-640) than the titers obtained by Wong et al. with $7.5 \mu \mathrm{g}$ HA (i.e., $\log _{10} 2.2-2.7$ or $\left.160-500\right)$. In contrast to undetectable VN titers in their study, our CaPNP-H5N1 vaccine could induce nearly 5-fold higher VN titers compared to non-adjuvanted vaccine (see Table IV). In a recently published and more relevant study by Nurpeisova et al. (47), mice were immunized twice with an inactivated whole virus $\mathrm{H} 5 \mathrm{~N} 1$ vaccine candidate containing 2.5-10 $\mu \mathrm{g} \mathrm{HA} /$ dose and alum as adjuvant. Antibody responses in sera were determined by HAI and VN titers post last immunization. The group of mice receiving $5 \mu \mathrm{g}$ or $10 \mu \mathrm{g}$ HA showed HAI titers of approximately 92 and 280 and VN titers of 280 and 420, respectively. All mice immunized with 5-10 $\mu \mathrm{g}$ HA survived the virus challenge with $10 \mu \mathrm{g}$ HA elicited the most protection from the disease. However, not all mice who received $2.5 \mu \mathrm{g}$ HA (HAI titers of about 60) survived and they also showed high virus titers in the lungs. Considering the significantly higher

Table III. Acute Toxicity/Inflammatory Response to CaPNP Administered by i.m. (1.2 mg/kg)

\begin{tabular}{|c|c|c|c|c|}
\hline & $\begin{array}{l}\text { Male/control } \\
(\text { mean } \pm \mathrm{SD})\end{array}$ & $\begin{array}{l}\text { Male/CaPNP } \\
(\text { mean } \pm \text { SD) }\end{array}$ & $\begin{array}{l}\text { Female/control } \\
(\text { mean } \pm \mathrm{SD})\end{array}$ & $\begin{array}{l}\text { Female/CaPNP } \\
(\text { mean } \pm \mathrm{SD})\end{array}$ \\
\hline Clinical observation (days 1-14) & Normal & Normal & Normal & Normal \\
\hline Weight gain $(\mathrm{g})$ & $122 \pm 24$ & $118+22$ & $92 \pm 17$ & $87 \pm 21$ \\
\hline Gross terminal necroscopy ${ }^{\mathrm{a}}$ & $0 / 5$ & $0 / 5$ & $0 / 5$ & $1 / 5^{\mathrm{b}}$ \\
\hline \multicolumn{5}{|c|}{ Skeletal muscle (hind limb) histopathology: } \\
\hline Inflammation, granulamotus & $0 / 5$ & $3 / 5$ & $0 / 5$ & $3 / 5$ \\
\hline$\left(\right.$ Severity score in parenthesis) ${ }^{c}$ & $(0)$ & $(1.15)$ & $(0)$ & $(0.75)$ \\
\hline
\end{tabular}

\footnotetext{
${ }^{a}$ Spleen, extra-orbital lacrimal gland. Salivary gland(s), mandibular lymph node (left) from 5 male +5 female animals

${ }^{b}$ Mottled slight pigmentation in one animal in salivary gland-considered incidental

${ }^{c}$ Mean group severity score relative to control $(0=$ none, $1=$ minimal, $2=$ mild, $3=$ moderate, and $4=$ marked $)$
} 
Table IV. A/H5N1/Vietnam/1203/2004-Derived HA(H5N1) Subunit Vaccine Immunogenicity in Mice After Two i.m. Immunization with $3 \mu \mathrm{g}$ HA (Titers at Week 10 Post 1st Immunization)

\begin{tabular}{|c|c|c|c|c|c|c|}
\hline Vaccine & HA/dose $(\mu \mathrm{g})$ & Anti-HA IgG titer (GMT) & $\mathrm{HAI}^{\mathrm{a}}(\mathrm{GMT})$ & $\log _{10} \mathrm{HAI}$ & $\mathrm{ND}_{50} / \mathrm{ml}^{\mathrm{b}}$ & $\operatorname{LogND}_{50} / \mathrm{ml}$ \\
\hline $\mathrm{HA}+0.06 \% \mathrm{CaP}$ & 3 & $1.31 \mathrm{E}+05$ & 320 & 2.5 & $3.62 \mathrm{E}+04$ & 4.56 \\
\hline $\mathrm{HA}+0.24 \% \mathrm{CaP}$ & 3 & $2.62 \mathrm{E}+05$ & 640 & 2.8 & $9.56 \mathrm{E}+04$ & 5.98 \\
\hline HA only & 3 & $8.65 E+04$ & 160 & 2.2 & $2.08 \mathrm{E}+04$ & 4.32 \\
\hline
\end{tabular}

${ }^{a}$ Values represent geometric mean HAI antibody titers and are expressed as the reciprocal of the highest dilution of serum inhibiting agglutination of $1 \%$ horse erythrocytes at 8 HA units of the VN1203 virus. Serum samples from 10 mice per group were collected on day 70 (week 10) after the first vaccinations

${ }^{b}$ The $50 \%$ neutralization dose $\mathrm{ND}_{50}$ is defined as the concentration of anti-virus antibody that reduces the number of infected cells by $50 \%$. $\mathrm{ND}_{50}$ was calculated by Karber formula

HAI and VN titers that we obtained in this study and in previous studies by us and others $(25,26,29-31)$, it is reasonable to suggest that CaPNP is a more effective adjuvant than alum and could provide full protection against $\mathrm{H} 5 \mathrm{~N} 1$ infection while allowing dose sparing at the same time. For instance, in our pandemic H1N1 vaccine study, while alum-adjuvanted or non-adjuvanted vaccines provided only $45-65 \%$ protection in mice, the vaccine containing CaPNP protected $>80 \%$ of animals from a lethal dose of $\mathrm{H} 5 \mathrm{~N} 1$ virus infection (25). We currently lack any preclinical studies to make a comparison between CaPNP and MF59 or A0S3. However, it is important to highlight that CaPNP presents significant advantages over most other approved or investigational adjuvants, including the emulsion-based adjuvants, with respect to its unique physicochemical properties (e.g., stable at storage under extreme temperature conditions, it can be autoclaved, lyophilized or spray-dried as free-flowing powder) and its inherently safe-nontoxic-biocompatible nature. CaPNP is also a single-component adjuvant which is one of the most desirable properties from any new adjuvant. Our manufacturing process is simple, reproducible, involves only a single step, and it has been already scaled up under GMP to manufacture multiple kilogram quantities of particles. In contrast, manufacturing of emulsion adjuvants comprises multi-step complex protocols and specialized equipment. For instance, formulation of MF59 requires combination of immiscible components (squalene and water); thus, the emulsion needs to be stabilized by surfactants or emulsifiers requiring high-speed mixing and processing through a microfluidizer to obtain uniform droplets of $160 \mathrm{~nm}$ (41). In contracts, CaPNP manufacturing does not involve any postprocess step or equipment to obtain uniformly distributed stable particles. Unlike MF59, our process has the flexibility to manufacture particles in any size in the $<100$ to $>1000 \mathrm{~nm}$ ranges with simple manipulation in process parameters. Safety expectations for adjuvants, particularly the ones used in influenza vaccines, have prime importance and critical from the regulatory and compliance standpoints. The benefits of including an adjuvant in vaccine must be weighed against the risk of adjuvant itself inducing serious local or systemic adverse reactions. For instance, safety concerns have been raised against AS03 after the reports of increased incidence of narcolepsy and risk of anaphylaxis in children vaccinated with AS03-adjuvanted $\mathrm{A}(\mathrm{H} 1 \mathrm{~N} 1 \mathrm{pdm})$ vaccine during the 2009 pandemic $(48,49)$. Given the likelihood of expanded use of adjuvanted vaccines against influenza infections, development of better/safer adjuvants that can positively influence both the magnitude and the pattern of the immune response to vaccination are highly relevant.

In this study, we also demonstrated preclinical acute safety of CaPNPs for administration via the IM route. Although the CaPNP-H5N1 vaccine study presented here was conducted in mice, we used albino guinea pigs to confirm that CaPNPs does not induce any systemic or local allergic reaction or inflammatory responses. Although a new compound or drug that shows safety in animal models may show very different safety profiles when administered to humans, using the most appropriate small animal model during early stages of toxicity testing is still very important and is an economical strategy. We justify the choice of guinea pig model for the following reasons. Guinea pig has been confidently used in pharmaceutical research for over 200 years. Albino Guinea pig is accepted as a highly sensitive animal model for toxicology studies, the most commonly used animal model to study infectious diseases (50), and for preclinical evaluation of vaccine adjuvants (51). The species and the number of animals employed in our protocol are in compliance with standard and excepted testing procedures as set forth in the Code of Federal Regulations (part 58 of Title 21). In addition, the dose route (i.e., IM) we used in toxicity testing is relevant to our influenza vaccine studies and corresponds to the potential route for H5N1 vaccination in human. In veterinary vaccines, there is no defined maximum limit for the adjuvant dose, which is often defined based on the balance between the vaccine efficacy and local reactogenicity. Whereas, there are limitations in the amount of adjuvants used in human vaccines. For natural calcium phosphate mineral adjuvant used in human vaccines in Europe for many decades (52), the limit was set as $1.30 \mathrm{mg}$ elemental $\mathrm{Ca}^{++}$per vaccine dose (53). That roughly translates to about $20 \mu \mathrm{g} \mathrm{Ca}++/$ $\mathrm{kg}$ body weight for a human subject with an average body weight of 70-75 kg. Our particles are not obtained from naturally mined calcium mineral but are synthetically manufactured as inorganic nanoparticles using proprietary processes. In our acute toxicity study, we administered guinea pigs with $1.2 \mathrm{mg} \mathrm{CaPNP} / \mathrm{kg}$ body weight. That corresponds to approximately $135 \mu \mathrm{g} \mathrm{Ca}^{++} / \mathrm{kg}$ which is nearly 8 -fold of the dose permitted in human vaccines. Thus, our data showed that CaPNP does not indicate any systemic toxicity, systemic inflammatory responses, or any adverse effects, even at $8 \times$ higher dose than permitted in human vaccines. On the other hand, there was a minimal or insignificant local inflammatory response observed in muscle tissues of some animals (severity score in the $0.75-1$ range). We speculate that a minimal inflammation in the local tissue with no clinical indication or 
systemic adverse effect may even be an advantage for influenza vaccine effectiveness. This may also suggest localized presence of residual CaPNP at the injection site recruiting macrophages and other immune cells. In other words, slow elimination of CaPNP from the injection site could be an advantage facilitating slow release of influenza antigen for uptake and presentation by the macrophages. No other CaPNP-related lesions were observed in any of the test animals.

In summary, we present here encouraging data to endorse CaPNP as a safe and an attractive vaccine adjuvant and delivery system for development of safe and potent H5N1 vaccines. Although preliminary, the current study also provided further confirmation to our previous studies demonstrating that CaPNP is capable of inducing high antigen-specific antibody titers and virus-specific HAI and VN titers when combined into influenza vaccines. Thus, it suggests ability to maximize vaccine performance and manufacturing capacity during an influenza pandemic. Availability of a single-component, non-toxic, biocompatible, and effective vaccine adjuvant would be critical to address any regulatory and public concerns for its use in pandemic emergencies. Further efforts are required to investigate the adjuvant effects of CaPNP on vaccine efficacy in comparison to currently approved influenza vaccine adjuvants.

\section{ACKNOWLEDGMENTS}

This study was supported by BioSante Pharmaceuticals Inc. and by CaPtivate Pharmaceuticals LLC. The authors thank BioCon Inc. (Rockville, MD) small animal facility staff for performing the animal studies, Battelle scientists for performing HAI and MN assays, and IITRI facility staff and scientists for performing the acute toxicity studies.

\section{COMPLIANCE WITH ETHICAL STANDARDS}

Ethics Statement All immunization studies were performed at BioCon Inc. (Rockville, MD) in the company's Association for Assessment and Accreditation of Laboratory Animal Care International (AAALAC)-accredited Laboratory Animal Research Facility. Animals were housed according to Office of Laboratory Animal Welfare (OLAW) and AAALAC Guidelines. All animal experiments and related protocols were reviewed and approved by the Institutional Animal Care and Use Committee (IACUC) of BioCon. Experiments were conducted in accordance with the guidelines set out by the AAALAC. Acute toxicity studies were performed separately with contract to IIT Research Institute (IITRI-Chicago, IL.) and in accordance with U.S. Food and Drug Administration (FDA) Good Laboratory Practice (GLP) Regulations as set forth in the Code of Federal Regulations.

Conflict of Interest The authors declare that they have no conflict of interest.

\section{PUBLISHER'S NOTE}

Springer Nature remains neutral with regard to jurisdictional claims in published maps and institutional affiliations.

\section{REFERENCES}

1. WHO consultation and information meeting on the composition of influenza virus vaccines for the northern hemisphere 20162017. Available at: www.who.int/influenza/vaccines/virus/recommendations/consultation201602/en/index2.html

2. NIAID Strategic Plan for Research on Vaccine Adjuvants. Available at: https://rsc.niaid.nih.gov/sites/default/files/NIAIDStrategicPlanVaccineAdjuvants.pdf

3. WHO, Cumulative number of confirmed human cases for H5N1, 2003-2017 http://www.who.int/influenza/ human_animal_interface/2017_06_15_tableH5N1corrected.pdf?ua $=1$

4. Taubenberger JK, Morens DM, Fauci AS. The next influenza pandemic: can it be predicted? JAMA. 2007;297:2025-7.

5. Ungchusak K, Auewarakul P, Dowell SF, Kitphati R, Auwanit W, Puthavathana P, et al. Probable person-to-person transmission of avian influenza a (H5N1). N Engl J Med. 2005;352:333-40.

6. He F, Leyrer S, Kwang J. Strategies towards universal pandemic influenza vaccines. Expert Rev Vaccines. 2016;15:215-25.

7. Editorial-The Lancet. Plotting a route to a universal influenza vaccine. Lancet Infect Dis 2018;18: 475.

8. Wheatley AK, Kent SJ. Prospects for antibody-based universal influenza vaccines in the context of widespread pre-existing immunity. Expert Rev Vaccines. 2015;14:1227-39.

9. Quinones-Parra S, Loh L, Brown LE, Kedzierska K, Valkenburg SA. Universal immunity to influenza must outwit immune evasion. Front Microbiol. 2014;5:Article 285.

10. WHO, "Global pandemic influenza action plan to increase vaccine supply," Tech. Rep. WHO/IVB/06.13; WHO/CDS/EPR/ GIP/2006.1, WHO, Geneva, 2006, http://whqlibdoc.who.int/hq/ 2006/WHO_IVB_06.13_eng.pdf?ua=1

11. Treanor JJ, Campbell JD, Zangwill KM, Rowe T, Wolff M. Safety and immunogenicity of an inactivated subvirion influenza a (H5N1) vaccine. N Engl J Med. 2006;354:1343-51.

12. Treanor JJ, Wilkinson BE, Masseoud F, Hu-Primmer J, Battaglia R, O'Brien D, et al. Safety and immunogenicity of a recombinant hemagglutinin vaccine for H5 influenza in humans. Vaccine. 2001;19:1732-7.

13. HHS, Office of ASPR, Medical Countermeasures (MCM). Pandemic Influenza: Current vaccine and adjuvant stockpiles. Available at: https://www.medicalcountermeasures.gov/media/ 35695/cioce_current_vaccine_and_adjuvant_stockpiles.pdf

14. Lewis DB. Avian fl u to human infl uenza. Annu Rev Med. 2006;57:139-54.

15. Haque A, Hober D, Kasper LH. Confronting potential influenza a (H5N1) pandemic with better vaccines. Emerg Infect Dis. $2007 ; 13: 1512-8$.

16. Vesikari T, Knuf M, Wutzler P, Karvonen A, Kieninger-Baum D, Schmitt HJ, et al. Oil-in-water emulsion adjuvant with influenza vaccine in young children. $N$ Engl J Med. 2011;365:1406-16.

17. Vesikari T, Kirstein J, Devota Go G, Leav B, Ruzycky ME, Isakov L, et al. Efficacy, immunogenicity, and safety evaluation of an MF59-adjuvanted quadrivalent influenza virus vaccine compared with non-adjuvanted influenza vaccine in children: a multicentre, randomised controlled, observer-blinded, phase 3 trial. Lancet Respir Med. 2018;6:345-56.

18. van den Brand JMA, Kreijtz JHCM, Bodewes R, Stittelaar KJ, van Amerongen G, Kuiken T, et al. Efficacy of vaccination with different combinations of MF59-Adjuvanted and Nonadjuvanted seasonal and pandemic influenza vaccines against pandemic H1N1 (2009) influenza virus infection in ferrets. J Virology. 2011;85:2851-8.

19. European Centre for Disease Prevention and Control. Narcolepsy in association with pandemic influenza vaccination (a multi-country European epidemiological investigation) Stockholm: ECDC; September 2012, Stockholm, Sweden, ISBN 97892-9193-388-4. (VAESCO report).

20. Reilly J. Final Report of National Narcolepsy Study Steering Committee, Investigation of an increase in the incidence of narcolepsy in children and adolescents in 2009 and 2010. April 
476 19, 2012. Available at: https://health.gov.ie/wp-content/ u p 1 o a d s / $2014 / 03 /$

Final_Report_of_National_Narcolepsy_Study_Steering_Committee.pdf. Accessed February 9, 2015 and Jan 6, 2019.

21. Dauvilliers Y, Arnulf I, Lecendreux M, Monaca Charley C, Franco P, Drouot X. Et a. Narcoflu-VF study group. Increased risk of narcolepsy in children and adults after pandemic H1N1 vaccination in France. Brain. 2013;136:2486-96.

22. Baudner BC, Ronconi V, Casini D, Tortoli M, Kazzaz J, Singh M, et al. MF59 emulsion is an effective delivery system for a synthetic TLR4 agonist (E6020). Pharm Res. 2009;26:1477-85.

23. Iyer V, Cayatte C, Guzman B, Schneider-Ohrum K, Matuszak $\mathrm{R}$, Snell A, et al. Impact of formulation and particle size on stability and immunogenicity of oil-in-water emulsion adjuvants. Hum Vaccin Immunother. 2015;11:1853-64.

24. Boraschi D, Italiani P. From antigen delivery system to Adjuvanticy: the board application of nanoparticles in vaccinology. Vaccines (Basel). 2015;3:930-9.

25. Morç 1 T, Hurst BL, Tarbet EB. Calcium phosphate nanoparticle (CaPNP) for dose-sparing of inactivated whole virus pandemic influenza a (H1N1) 2009 vaccine in mice. Vaccine. 2017;35:4569-77.

26. Joyappa DH, Kumar CA, Banumathi N, Reddy GR, Suryanarayana VVS. Calcium phosphate nanoparticle prepared with foot and mouth disease virus P1-3CD gene construct protects mice and Guinea pigs against the challenge virus. Vet Microbiol. 2009;139:58-66.

27. He Q, Mitchell A, Johnson SL, Wagner-Bartak C, Morcol T, Bell SJD. Calcium phosphate nanoparticle adjuvant. Clin Diagn Lab Immunol. 2000;6:899-903.

28. He Q, Mitchell AR, Johnson SL, Morcol T, Bell SJD. Calcium phosphate particles induced mucosal immunity and protection against herpes simplex V2. Clin Diagnos Lab Immunol. 2002;9:1021-4.

29. Olmedo H, Herrera M, Rojas L, et al. Comparison of the adjuvant activity of aluminum hydroxide and calcium phosphate on the antibody response towards Bothrops asper snake venom. J Immunotoxicol. 2013;11:44-9.

30. Koppad S, Raj GD, Gopinath VP, Kirubaharan JJ, Thangavelu A, Thiagarajan V. Calcium phosphate coupled Newcastle disease vaccine elicits humoral and cell mediated immune responses in chickens. Res Vet Sci. 2011;91:384-90.

31. Amini Y, Moradi B, Tafaghodi M, Meshkat Z, Ghazvini K, Fasihi-Ramandi MTB. Trifusion antigen adsorbed on calcium phosphate nanoparticles stimulates strong cellular immunity in mice. Biotechnol Bioprocess Eng. 2016;21:653-8.

32. Karam HM, Shaaban EA, Mohamed AF, Zaki HF, Kenawy SA. New approach for improving production of Naja Haje snake antivenom. Int J Science Res Publications. 2015;XXXX:5.

33. CaPtivate Pharmaceuticals Reports Data from Influenza A/ H1N1 2009 Pandemic virus vaccine studies funded by NIAID/ $\mathrm{NIH}$ preclinical services program: $\mathrm{CaP}$ nanoparticle adjuvant significantly improves vaccine efficacy. Available at: http:// www.ireachcontent.com/news-releases/captivate-pharmaceuticals-reports-data-from-influenza-ah1n1-2009-pandemic-virusvaccine-studies-funded-by-niaidnih-preclinical-services-program-671631134.html

34. Mor ol T, Weidner JM, Mehta A, Bell SJD, Block T. Calcium phosphate particles as pulmonary delivery system for interferon- $\alpha$ in mice. AAPS PharmSciTech. 2018;19:395-412.

35. Morçol T, Nagappan P, Nerenbaum L, Mitchell A, Bell, SJD. (2006) Particulate Drug Delivery Systems for Protein Drugs: An Overview of Non-invasive Insulin Delivery Using Calcium Phosphate Particle Technology in: Handbook of Particulate Drug Delivery, Chapter 11, Kumar, R.M.N.V. (Ed.), American Scientific Publishers, Stevenson Ranch, CA.
36. Banchereau J, Briere F, Caux C, Davoust J, Lebecque S, Liu YJ, et al. Immunobiology of dendritic cells. Annu Rev Immunol. 2000;18:767-811.

37. Huang X, Karabudak A, Comber J, Morcol T, Philip R. Novel vaccination approach for dengue infection based on conserved $\mathrm{T}$ cell epitopes formulated in calcium phosphate nanoparticles. Human Vaccines Immothera. 2017;13:2612-25. https://doi.org/ 10.1080/21645515.2017.1369639.

38. O'Hagan DT, Tsai T, Reed S. Emulsion based adjuvants for improved influenza vaccine in: influenza vaccines for the future. Del Giudice G, Rappuoli R (Eds). Springer; Basel AG. In: 327358; 2011

39. O'Hagan DT, Ott GS, De Gregorio E, Seubert A. The mechanism of action of MF59 - an innately attractive adjuvant formulation. Vaccine. 2012;30:4341-8.

40. Leroux-Roels I, Roman F, Forgus S, Maes C, de Boever F, Dramé M, et al. Priming with AS03-adjuvanted H5N1 influenza vaccine improves the kinetics, magnitude and durability of the immune response after a heterologous booster vaccination: an open non-randomized extension of a double-blind randomized primary study. Vaccine. 2010;28:849-57.

41. O'Hagan DT, Ott GS, Nest GV, Rappuoli R. DelGiudice. The history of MF59® adjuvant: a phoenix that arose from the ashes. Expert Rev Vaccines. 2013;12:13-30.

42. Seasonal Flu Vaccine Fluad Approved by FDA. Deceber 02, 2015. Available at: https://www.pharmacytimes.com/productnews/seasonal-flu-vaccine-fluad-approved-by-fda.

43. BioSante Pharmaceuticals, Inc. Announces CaP Nanotechnology Product Development-New Product. February 5, 2004. Available at: https://www.azonano.com/ article.aspx?ArticleID $=477$

44. Ramakrishnan MA. Determination of $50 \%$ endpoint titer using a simple formula. World J Virol. 2016;5:85-6.

45. Lu X, Tumpey TM, Morken T, Zaki SR, Cox NJ, Katz JM. A mouse model for the evaluation of pathogenesis and immunity to influenza a (H5N1) viruses isolated from humans. J Virology. 1999;73:5903-11.

46. Wong S-S, DeBeauchamp J, Zanin M, Sun Y, Tang J, Webby R. H5N1 influenza vaccine induces a less robust neutralizing antibody response than seasonal trivalent and H7N9 influenza vaccines. NPJ Vaccines. 2017;16:16. https://doi.org/10.1038/ s41541-017-0017-5.

47. Nurpeisova A, Kassenov M, Rametov N, Tabynov K, Renukaradhya GJ, Volgin Y, et al. Analysis of the efficacy of an adjuvant-based inactivated pandemic H5N1 influenza virus vaccine. Arch Virol. 2019;164:1027-36.

48. Nohynek H, Jokinen J, Partinen M, Vaarala O, Kirjavainen T, Sundman J. AS03 adjuvanted AH1N1 vaccine associated with an abrupt increase in the incidence of childhood narcolepsy in Finland. PLoS One. 2012;7:e33536.

49. Rouleau I, De Serres G, Drolet JP, Skowronski DM, Ouakki M, Toth E. Increased risk of anaphylaxis following administration of 2009 AS03-adjuvanted monovalent pandemic a/H1N1 (H1N1pdm09) vaccine. Vaccine. 2013;31:5989-96.

50. Padilla-Carlin DJ, McMurray DN, Hickey AJ. The Guinea pig as a model of infectious diseases. Comp Med. 2008;58:324-40.

51. Hogarth PJ, Jahans KJ, Hecker R, Hewinson RG, Chambers MA. Evaluation of adjuvants for protein vaccines against tuberculosis in Guinea pigs. Vaccine. 2013;21:977-82.

52. Relyveld EH. Preparation and use of calcium phosphate adsorbed vaccines. Dev Biol Stand. 1986;65:131-6.

53. Ph.Eur. 6.ed. Vaccines for human use, pp. In: 3971; 2009.

Publisher's Note Springer Nature remains neutral with regard to jurisdictional claims in published maps and institutional affiliations. 